\title{
Irisin Exerts Neuroprotective Effects on Cultured Neurons by Regulating Astrocytes
}

\author{
Kexin Wang, ${ }^{1}$ Hongyan Li, ${ }^{2}$ Hongxing Wang, ${ }^{3}$ Jun-hui Wang $\mathbb{D},{ }^{4}$ Feng Song $\left(\mathbb{D},{ }^{5}\right.$ \\ and Yu Sun iD ${ }^{6}$ \\ ${ }^{1}$ Department of General Surgery, Qilu Hospital of Shandong University, Jinan, Shandong, China \\ ${ }^{2}$ Department of Obstetrics and Gynecology, The Eighth People's Hospital of Qingdao, Qingdao, Shandong, China \\ ${ }^{3}$ Department of Neurology, Xuanwu Hospital, Capital Medical University, Beijing, China \\ ${ }^{4}$ Department of Physiology, University of Toronto, Toronto, Ontario, Canada \\ ${ }^{5}$ Department of Orthopedics, Qingdao University Affiliated Qingdao Municipal Hospital, Qingdao, Shandong, China \\ ${ }^{6}$ Department of Endocrinology, Qilu Hospital of Shandong University, Jinan, Shandong, China
}

Correspondence should be addressed to Feng Song; songfengqd@gmail.com and Yu Sun; sunyujinan@gmail.com

Received 14 June 2018; Revised 12 August 2018; Accepted 27 August 2018; Published 26 September 2018

Academic Editor: Catherine Lawrence

Copyright (C) 2018 Kexin Wang et al. This is an open access article distributed under the Creative Commons Attribution License, which permits unrestricted use, distribution, and reproduction in any medium, provided the original work is properly cited.

\begin{abstract}
Neurons suffer detrimental effects from $\beta$-amyloid toxicity in Alzheimer's disease. The exercise hormone, irisin, is found to induce a neuroprotective gene program and facilitates the beneficial effects on cognitive function. But no effort is made to test its direct protective effects on neurons against the $\mathrm{A} \beta$-induced cell toxicity so far. In the present study, we investigated whether irisin could protect neurons against A $\beta$ - (25-35) induced cell damage and explored the possible underlying mechanisms. Primary cell cultures of astrocytes and neurons were established. Conditioned medium from astrocyte was collected for the treatment and biochemistry assay study. To explore the protein expression changes, Western blot and ELISA assays were used in these in vitro cell culture models. Exposure of hippocampal neurons to $10 \mu \mathrm{M} \mathrm{A} \beta$ (25-35) caused significant reduction on cell viability, and the toxic effect was not significantly reduced by the coadministration of irisin. However, pretreated astrocyte-conditioned medium with irisin for 12 hours notably protected the neurons from the toxicity of $\mathrm{A} \beta$. Also, we found that irisin could attenuate the release of IL- 6 and IL- $1 \beta$ from cultured astrocytes and decrease the expression level of COX-2 and phosphorylation of AKT. Last, we found that irisin could reduce NFkB activation in astrocyte exposed to A $\beta$ by preventing the phosphorylation and the loss of $\mathrm{I} \kappa \mathrm{B} \alpha$. Our finding may provide novel evidence for the future application of irisin in the treatment of Alzheimer's disease and the memory dysfunction in diabetes mellitus.
\end{abstract}

\section{Introduction}

Alzheimer's disease $(\mathrm{AD})$ is a progressive neurodegenerative disorder, which is characterized by extracellular amyloid plaques and cytoplasmic tau tangles [1]. Although progress has been made of understanding this devastating disease during the recent decade, $\mathrm{AD}$ remains incurable and is still a major healthcare challenge for medical communities globally.

Currently, many recent findings implicate soluble $\beta$ amyloid $(\mathrm{A} \beta \mathrm{s})$ but not the fibrillar aggregates contribute to the pathogenesis of $\mathrm{AD}$. Soluble $\mathrm{A} \beta$ has demonstrated cytotoxic effects on neural cells, although the mechanism is not clear so far [2]. Proinflammatory cytokines are considered the possible candidates that mediated the cytotoxic effects of $\mathrm{A} \beta$ [3]. The consequent neuronal injury of neuroinflammation derived from $\mathrm{A} \beta$ includes axonal cytoskeleton breakdown and axonal swellings as well [4]. In the central nervous system (CNS), astrocyte is one of the major players which are responsible for producing cytokines locally [5]. The interaction between astrocyte and neuron in $\mathrm{AD}$ mouse model has been proved to be essential part of the $\mathrm{AD}$ pathogenesis and a promising target of new drug treatment [6]. Therefore, exploring the role of astrocyte in the $A \beta$-induced neuronal impairment is becoming very attractive during the years.

Irisin, also known as FNDC5, is a myokine which can induce "browning" of white adipose tissue and increase energy expenditure [7]. Irisin could ameliorate glucose metabolic 
derangements and might be promising novel therapeutic compound for diabetes mellitus (DM) in the future. Statistical analysis has revealed that about $70 \%-80 \%$ of AD patients may have DM or abnormal glucose metabolism [8]. Moreover, irisin is an active mediator for the beneficial effects of exercise on the CNS [9], which suggests that irisin could play an important therapeutic role in some brain disorders. Also, no effort is made to examine whether irisin can protect neuronal cells against $\mathrm{A} \beta$-induced cytotoxicity.

In the present study, we want to investigate whether astrocyte is an active participator which mediates the neuroprotective effects of irisin. We found that irisin had no direct protective effects on neurons, but conditioned medium from irisin-pretreated astrocyte cultures could significantly attenuate the cytotoxicity of $A \beta$. Irisin was able to inhibit the release of IL- $1 \beta$ and IL- 6 from astrocyte in cultures. The beneficial effects of irisin in astrocyte might be through the inhibition of $\mathrm{NF} \kappa \mathrm{B}$ activation by reducing the phosphorylation of $\mathrm{I} \kappa \mathrm{B} \alpha$ and preventing the loss of total $\mathrm{I} \kappa \mathrm{B} \alpha$ in astrocyte exposed to $\mathrm{A} \beta$.

\section{Methods}

2.1. Primary Cells and Drug Treatments. Cerebral hippocampal astrocytes were isolated from postneonatal pups of C57BL/6J mice as previously described $[6,10]$. Cultures were incubated in a $95 \%$ humidified incubator at $37^{\circ} \mathrm{C}$ with $5 \%$ $\mathrm{CO}_{2}$. The astrocyte cultures were fed Dulbecco's modified Eagle's medium (DMEM, Life Technologies) with 10\% fetal bovine serum (FBS, Life Technologies) every 3 days till they were 4 weeks old [6]. Hippocampal neurons were prepared from 16- to 18-day embryonic C57BL/6J mice as previously reported [6]. Hippocampal neurons were used for experiments on days 12-14 [6]. Irisin and $\mathrm{A} \beta_{25-35}$ were purchased from Sigma and was dissolved in culture media. The doses of these drugs for treatment were supported from previous literatures $[11,12]$.

\subsection{Preparation of Astrocyte-Conditioned Medium (ACM).} Astrocyte cultures were incubated with irisin for 12 hours and then replaced with fresh medium. The ACM was collected 24 hours later. Control ACM (without irisin treatment) was collected with the same procedure. For the ACM treatment in neurons, $1 \mathrm{ml} \mathrm{ACM}$ and $1 \mathrm{ml}$ fresh medium $(1: 1)$ were added to the neuron cultures as suggested by a previous report [13].

2.3. Cell Viability Assay. MTT assay was used to measure cell viability according to a previous report [7]. For cell viability study, neurons were plated in 96-well plates $\left(2 \times 10^{5}\right.$ cells per well). MTT solution was added into the culture media with a final concentration of $10 \%$ in each well. After incubation for $4 \mathrm{~h}\left(37^{\circ} \mathrm{C}\right), 100 \mu \mathrm{l}$ of dimethylsulfoxide (DMSO) was added to each well for another $15 \mathrm{~min}$. Then, the absorbance values were determined with a microplate reader (Thermo Fisher Scientific, Waltham, MA) at $570 \mathrm{~nm}$.

2.4. Immunofluorescence Staining. Culture dishes were fixed with $4 \%$ paraformaldehyde for $30 \mathrm{~min}$ and then were treated with $0.2 \%$ Triton X-100 for another $30 \mathrm{~min}$. After blocking with $3 \%$ bovine serum albumin for $2 \mathrm{~h}$, primary antibodies to glial fibrillary acidic protein (GFAP) (Millipore, Burlington, MA) and microtubule-associated protein 2 (MAP2) (Millipore, Burlington, MA) were used overnight and followed by the detection of secondary antibodies (Invitrogen-Molecular Probes) for 2 hours. $1 \mu \mathrm{g} / \mathrm{ml}$ Hoechst 33342 (Invitrogen-Molecular Probes) was used for nuclear staining.

2.5. Western Blot. Protein was extracted from cultured cells with cold lysis buffer containing phosphatase and protease inhibitor cocktail (Sigma). Total proteins were separated by electrophoresis with the $12 \%$ SDS-PAGE and then transferred onto nitrocellulose membranes (Thermo Fisher Scientific, Waltham, MA). The target proteins were measured using the primary antibodies of COX-2 (Abcam, Cambridge, United Kingdom), phosphorylated AKT (p-AKT, Cell Signaling, Danvers, MA), total AKT (t-AKT, Cell Signaling, Danvers, MA), NFkB-p65 (Abcam, Cambridge, United Kingdom), phosphorylated $\mathrm{I} \kappa \mathrm{B} \alpha$ (p-I $\kappa \mathrm{B} \alpha$, New England Biolabs, Beverly, MA), total $\mathrm{I} \kappa \mathrm{B} \alpha$ (t-I $\kappa \mathrm{B} \alpha$, New England Biolabs, Beverly, MA), and followed by detection with the secondary antibody and ECL kit (PerkinElmer, Waltham, MA). $\beta$-Actin (Santa Cruz, Dallas, TX) antibodies were used here as an internal control. Quantitative results are expressed as a ratio of target proteins to their internal control and phosphorylated proteins to their total proteins, accordingly.

2.6. Enzyme-Linked Immunosorbent Assay (ELISA). The content of IL- $1 \beta$ and IL- 6 in astrocyte medium was measured by using ELISA kits (BosterBio, Pleasanton, CA), according to the manufacturer's manual. For the ELISA test, astrocytes were plated in 96-well plate (Sigma, Saint Louis, MO) instead of petri dishes. Each treatment contained at least 6 wells, and the total medium from all these wells was mixed together. $200 \mu \mathrm{l}$ medium was loaded for each well in ELISA plate, and each sample was assayed in triplicate.

2.7. Statistical Analysis. GraphPad PRISM 5.0 software (GraphPad Software, La Jolla, CA) was used in the present study for statistical analysis. Comparison between groups was performed using analysis of one-way ANOVA followed by Newman-Keuls post hoc test or Student's $t$-test. Data are presented as mean \pm S.E.M. Differences were considered statistically significant at $p<0.05$.

\section{Results}

3.1. Irisin Protected Neuron in Cultures against $A \beta$-Induced Cell Viability Loss by Modulating Astrocytes. First, we set up cultures of hippocampal astrocytes and neurons, as shown in Figure 1(a). The purity of these cultured cells was identified with specific markers of GFAP and MAP2 for astrocyte and neuron, respectively. And then we asked whether irisin could exert neuroprotective effects on these culture models. We found that $10 \mu \mathrm{M} \mathrm{A} \beta(25-35)$ treatment could cause significant loss of neuronal cell viability in 24 hours, but cotreatment of 5 or $10 \mathrm{nM}$ irisin could not rescue the cell loss (Figure 1(b)). Next, we tested whether astrocyte could mediate the protective effects of irisin on neurons. Astrocyte 

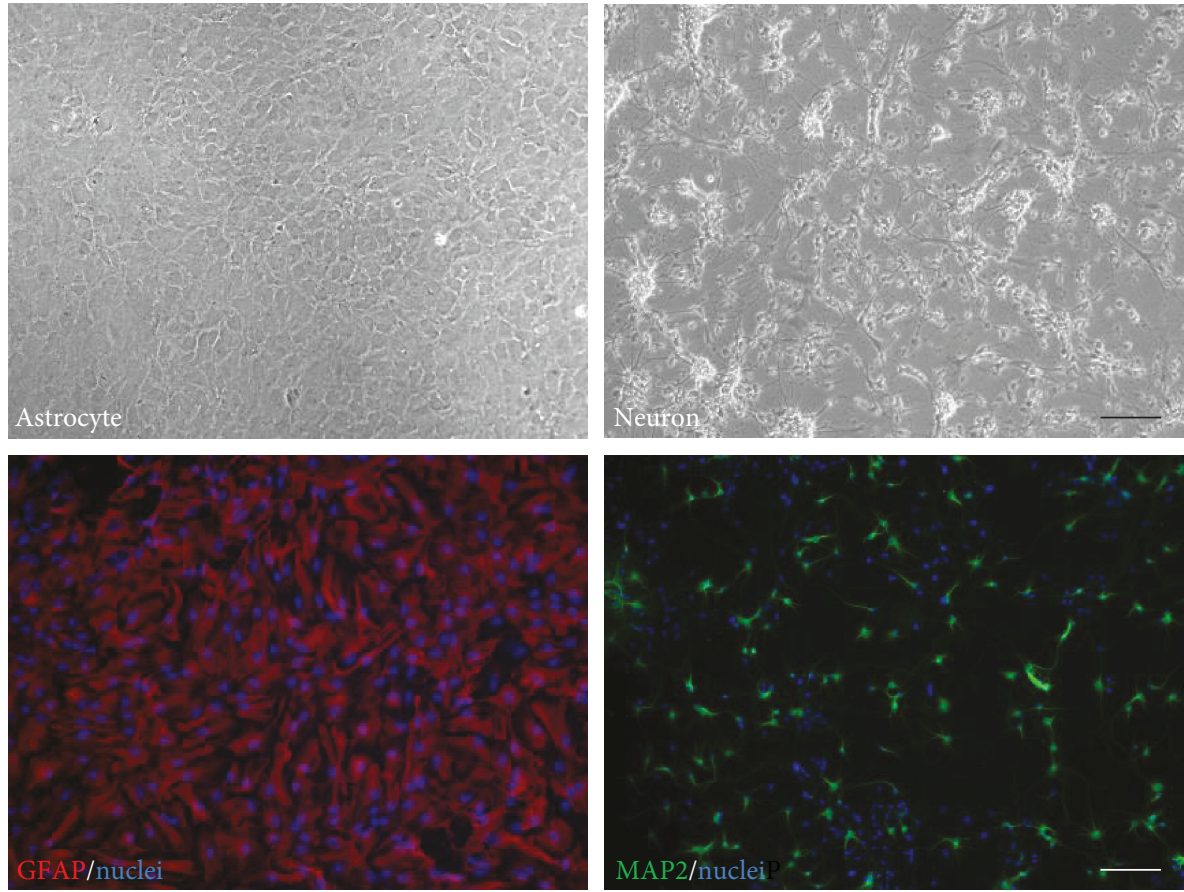

(a)

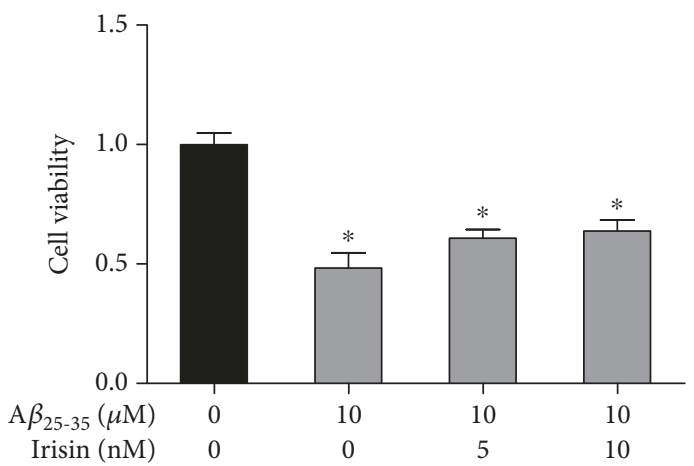

(b)

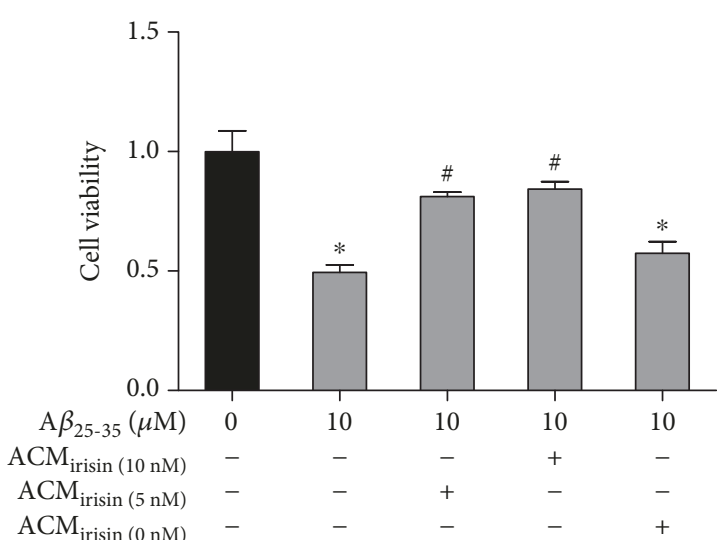

(c)

FIGURE 1: $\mathrm{A} \beta_{25-35}$ caused significant loss of cell viability in primary cultures of hippocampal neurons, which is not reversed by irisin cotreatment. (a) Representative phase contrast photographs of cultured hippocampal astrocytes ( 2 weeks old) and neurons ( 7 days). Representative immunofluorescent photographs of astrocyte markers GFAP and MAP2. Hoechst 33258 used as nuclei staining. Scale bar represents $100 \mu \mathrm{M}$. (b) Irisin had no direct protective effects on the cell viability in neuron exposed to A $\beta_{25-35}$ (MTT). (c) ACM irisin exerted protective effects on the cell viability in neuron exposed to $\mathrm{A} \beta_{25-35}$ while control ACM without pretreatment of irisin had no such protective effects (MTT). Data are expressed as means \pm SEM, ${ }^{*} p<0.05$ vs control, ${ }^{\#} p<0.05$ vs A $\beta_{25-35}, n=6$.

cultures were incubated with irisin $(0,5$, and $10 \mathrm{nM})$, and ACM was collected after 12 hours to incubate the hippocampal neurons for 30 minutes before $\mathrm{A} \beta_{25-35}$ was added. As shown in Figure 1(c), conditioned medium from irisin(5 and $10 \mathrm{nM}$ ) pretreated astrocytes significantly improved the cell viability of neurons exposed to $\mathrm{A} \beta_{25-35}$ while control ACM showed no significant effect on the cell viability. The above results implied that irisin might exert its neuroprotective effect by modulating astrocytes.

3.2. Irisin Reduced the Released IL-1 $\beta$ and IL-6 Level in Cultured Astrocyte Exposed to $A \beta_{25-35}$. Next, we hypothesized that irisin might inhibit the release of soluble factors which mediated the detrimental effects on neurons in cultures. Proinflammatory cytokines are a group of factors which are closely involved in the amyloid-induced cell injury in $\mathrm{AD}$ [14]. In the present study, we investigated two important cytokines, IL-1 $\beta$ and IL-6. ACM was collected after 12-hour irisin treatment, and ELISA experiments were carried out to measure the level of these two cytokines in the medium. As shown in Figure 2(a), $A \beta_{25-35}$ treatment resulted in a significant increase of released IL- $1 \beta$ level in astrocyte cultures, which could be effectively decreased with cotreatment of irisin. And then we investigated the effects of irisin on the 


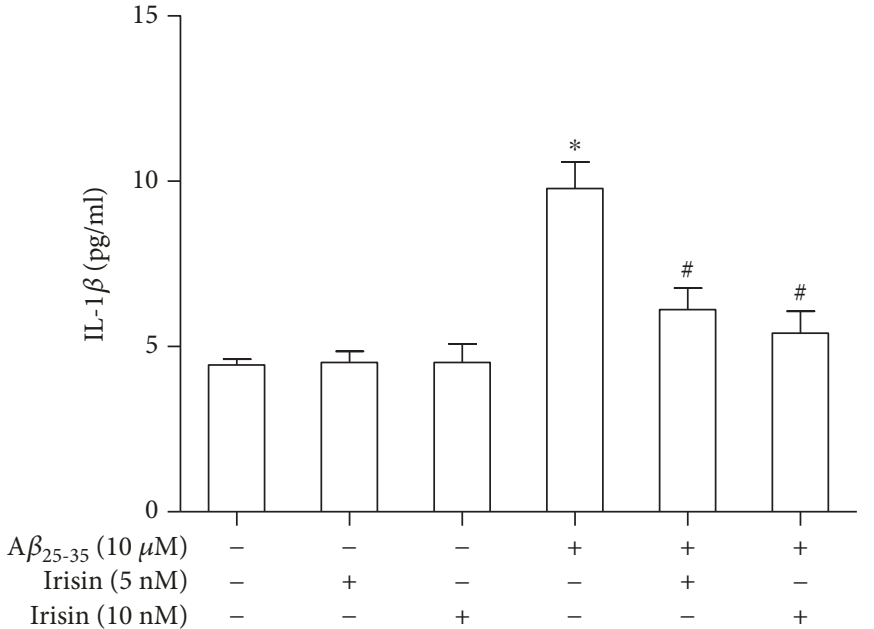

(a)

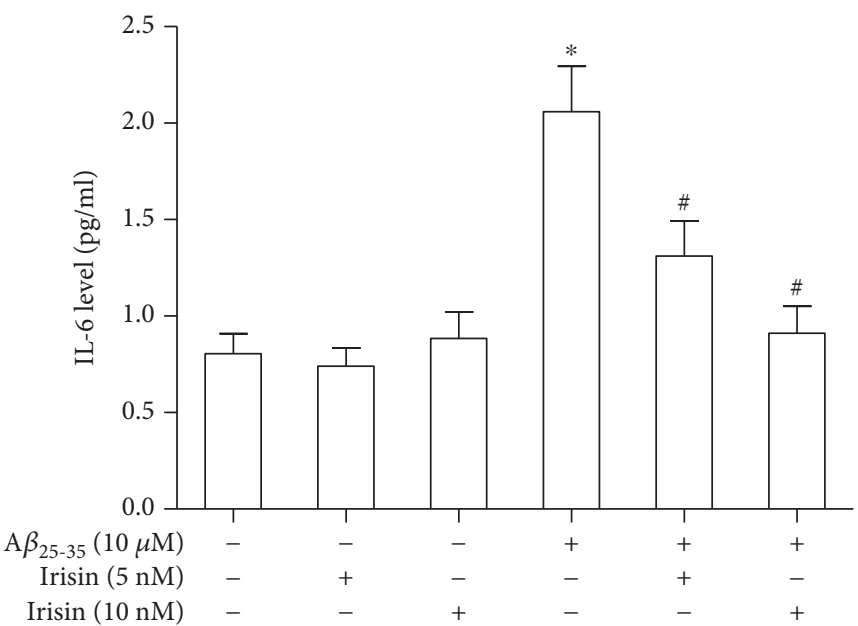

(b)

FIGURE 2: Irisin decreased the release of IL- $1 \beta$ and IL- 6 from cultured astrocytes exposed to A $\beta_{25-35}$. (a) Irisin could significantly prevent the $\mathrm{A} \beta_{25-35}$-induced IL- $1 \beta$ upregulation in ACM. (b) Irisin could significantly prevent the A $\beta_{25-35}$-induced IL-6 upregulation in ACM. Data are expressed as means \pm SEM, ${ }^{*} p<0.05$ vs control, ${ }^{\#} p<0.05$ vs A $\beta_{25-35}, n=5$.
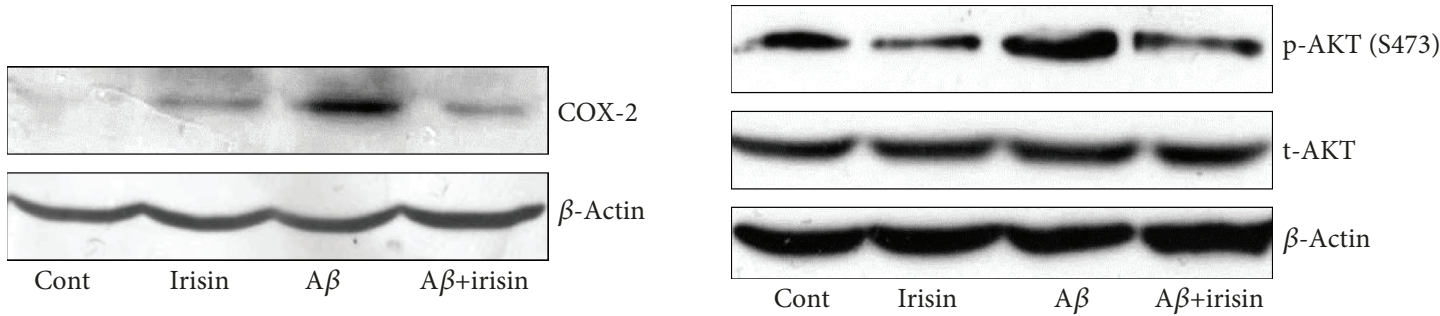

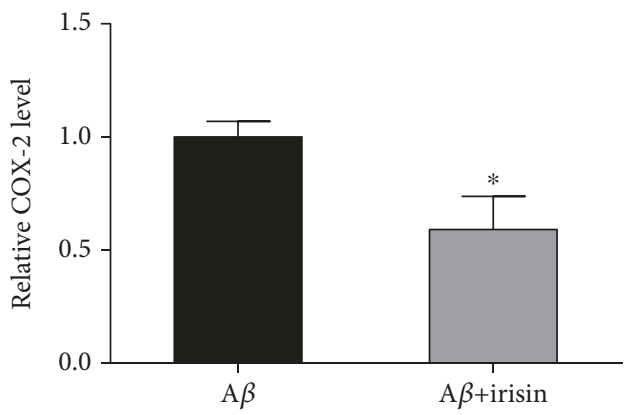

(a)

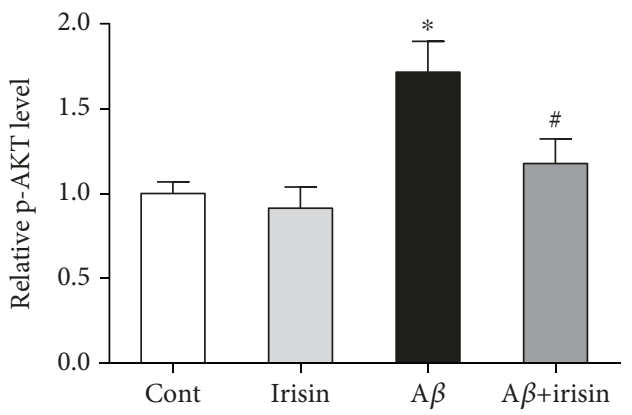

(b)

FIGURE 3: Irisin decreased the increased expression of COX-2 and p-AKT in cultured astrocytes exposed to A $\beta_{25-35}$. (a) Representative picture and statistical result showing irisin decreased the COX-2 level in astrocytes exposed to A $\beta_{25-35}$. (b) Representative picture and statistical result showing irisin decreased the $\mathrm{p}-\mathrm{AKT}$ level in astrocyte exposed to $\mathrm{A} \beta_{25-35}$. Data are expressed as means \pm SEM, ${ }^{*} p<0.05$ vs control, ${ }^{\#} p<0.05$ vs $\mathrm{A} \beta_{25-35}, n=5$.

release of IL- 6 in cultured astrocyte. We found that irisin could also obviously prevent the boost of released IL-6 level in astrocyte cultures exposed to $A \beta_{25-35}$. These results suggested that irisin might attenuate the $A \beta_{25-35}$-induced neuronal cell loss by inhibiting the release of IL- $1 \beta$ and IL-6 from astrocyte.

3.3. Irisin Reduced the Inducible Expression of Cyclooxygenase2 (COX-2) and Phosphorylation of AKT in Cultured Astrocyte
Exposed to $A \beta_{25-35}$. Induction of COX-2, a proinflammatory factor, is involved in inflammatory response [15]. We postulated that COX was a possible candidate protein which was regulated by irisin to attenuate the inflammation in astrocyte exposed to $\mathrm{A} \beta_{25-35}$.

As shown in Figure 3(a), COX-2 demonstrated an inducible expression in astrocyte treated with $\mathrm{A} \beta_{25-35}$, but the upregulation was prevented by cotreatment with irisin. Another key protein in inflammation, AKT, was investigated 

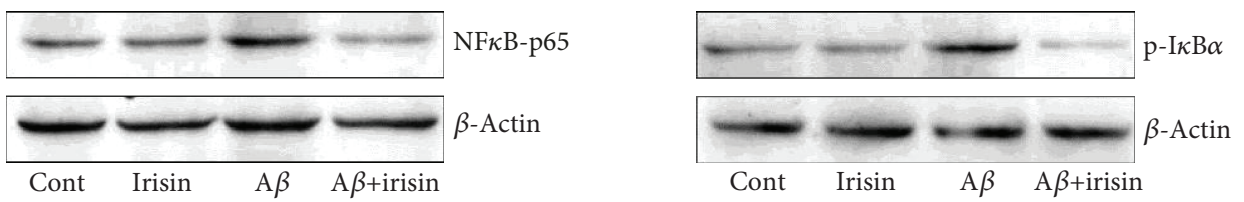

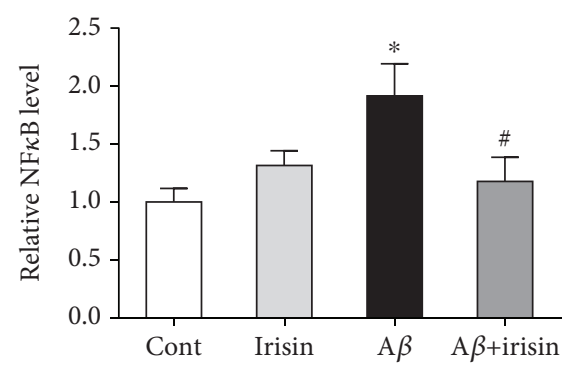

(a)

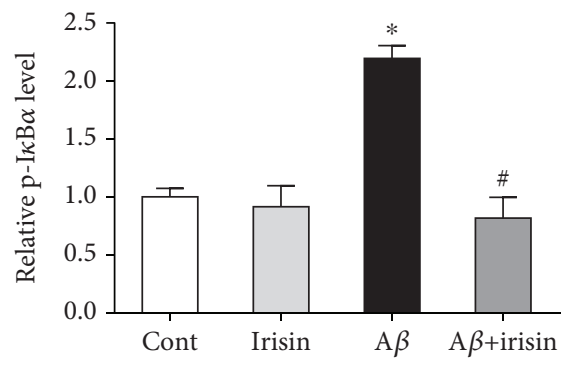

(b)
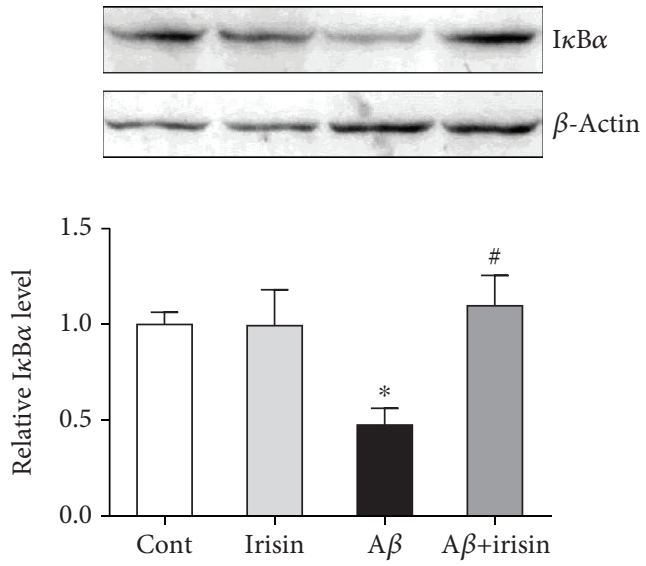

(c)

FIgURE 4: Irisin decreased $\mathrm{NF} \kappa \mathrm{B}$ activity by preventing the phosphorylation and loss of $\mathrm{I} \kappa \mathrm{B} \alpha$ in cultured astrocytes exposed to $\mathrm{A} \beta_{25-35}$. (a) Representative picture and statistical result showing irisin decreased the NFkB level in astrocytes exposed to A $\beta_{25-35}$. (b) Representative picture and statistical result showing irisin decreased the $\mathrm{p}-\mathrm{I} \kappa \mathrm{B} \alpha$ level in astrocytes exposed to $\mathrm{A} \beta_{25-35}$. (c) Representative picture and statistical result showing irisin increased the total $\mathrm{I} \kappa \mathrm{B} \alpha$ level in astrocytes exposed to $\mathrm{A} \beta_{25-35}$. Data are expressed as means $\pm \mathrm{SEM}$, ${ }^{*} p<0.05$ vs control, ${ }^{*} p<0.05$ vs $\mathrm{A} \beta_{25-35}, n=5$.

with Western blot to measure the phosphorylation of this protein in the following Western blot study. We found that p-AKT (S473) was significantly upregulated in cultured astrocytes with $\mathrm{A} \beta_{25-35}$ treatment compared to total AKT, which was able to be partially reversed by the cotreatment of irisin (Figure 3(b)).

\subsection{Irisin Decreased Phosphorylation and Degradation of} I $\kappa \alpha \alpha$ Leading to $N F \kappa B$ Lower Activation in Astrocyte. We further explored the possible mechanism by which irisin regulated the COX-2 and proinflammatory factors. We assessed the protein expression level of NFKB (p65) in astrocyte cultures, and our results showed that $\mathrm{NF} \kappa \mathrm{B}$ protein level was increased after $\mathrm{A} \beta_{25-35}$ treatment but irisin could effectively decrease the upregulated expression (Figure 4(a)). Moreover, the phosphorylation of $\mathrm{I} \kappa \mathrm{B} \alpha(\mathrm{p}-\mathrm{I} \kappa \mathrm{B} \alpha)$, a $\mathrm{NF} \kappa \mathrm{B}$ inhibitor protein, was also significantly upregulated in astrocyte after $\mathrm{A} \beta_{25-35}$ treatment although it could be prevented by irisin cotreatment (Figure 4(b)). Accordingly, total $\mathrm{I} \kappa \mathrm{B} \alpha$ protein expression level in cultured astrocytes was reduced
(Figure 4(c)). Collectively, these Western blot results implied that irisin not only directly decreased the $\mathrm{NF} \kappa \mathrm{B}$ expression level but also reduced the phosphorylation and the loss of total $\mathrm{I} \kappa \mathrm{B} \alpha$, which might prevent the release of $\mathrm{NF} \kappa \mathrm{B}$ from $\mathrm{I} \kappa \mathrm{B} \alpha$ in astrocyte and finally inhibit the level of cytokines released from astrocyte.

\section{Discussion}

$\mathrm{AD}$ is one of the most devastating diseases among dementia. Recent studies have supported the idea that soluble $\mathrm{A} \beta$ might be the culprit of neuronal cell injury and synaptic impairment in $\mathrm{AD}$ [16]. Reducing soluble $\mathrm{A} \beta$ rather than insoluble $\mathrm{A} \beta$ improved the memory and cognitive performance in transgenic mice of $\mathrm{AD}$ with plaques [17]. Therefore, preventing the soluble $\mathrm{A} \beta$ cytotoxicity to neurons may be another promising strategy to treat $\mathrm{AD}$.

In the present study, we carried out experiments on cultured astrocytes and neurons to investigate whether an exercise hormone could prevent the cytotoxicity of soluble 
$A \beta$. Astrocytes have been found to be a potential target for the development of neuroprotective drugs for many years [6]. Consistent with previous studies, we found that soluble A $\beta$ treatment could cause the loss of neuronal cell viability in cultures. To prevent cell injury, we introduced irisin into the cell culture model to see whether this myokine could protect neurons from $\mathrm{A} \beta$ injury. Our results showed that irisin exerted its protective effects on neurons via an astrocytedependent way. Irisin had no direct protective effects on cultured neurons but was able to significantly reduce the release of cytokines from astrocytes. Moreover, this resulted in notable neuroprotection of the astrocyte-conditioned medium on hippocampal neurons in cultures. This suggests that astrocytes are essential parts of the protective effects of irisin on neurons in $\mathrm{AD}$.

As mentioned above, cytokines have been found to play an important role in $A \beta$-induced neuropathology of $\mathrm{AD}$. As astrocytes are major producers of these cytokines within the CNS, we asked whether irisin regulated the release of cytokines from astrocytes during its protective effects on neurons. IL- $1 \beta$ and IL- 6 were investigated in the present study, and we found that irisin could significantly prevent the $\mathrm{A} \beta$-induced increase of these two cytokines of astrocyte culture medium. The reduction of IL- $1 \beta$ and IL- 6 might be responsible for the consequent beneficial effects of conditioned medium on neurons. Next, we tried to decipher the possible underlying mechanisms which are responsible for the above changes. COX-2 can be induced by proinflammatory stimuli in the CNS [18]. Therefore, we explored whether COX-2 and another important player in inflammation, AKT, were regulated during the treatment in cultures. As expected, our results demonstrated that irisin inhibited the expression level of these two proteins in astrocytes exposed to $A \beta$. Since the promoter area of COX-2 gene includes sequences that can be responsive to regulation by $\mathrm{NF} \kappa \mathrm{B}$ [19], we also tested whether $\mathrm{NF} \kappa \mathrm{B}$ was involved in the regulation. We found that this transcriptional factor was significantly upregulated by $\mathrm{A} \beta$ treatment but irisin cotreatment could effectively reduce the increased expression level in astrocytes. Since $\mathrm{I} \kappa \mathrm{B} \alpha$ could inhibit $\mathrm{NF} \kappa \mathrm{B}$ activity through sequestration in the cytoplasm [15], the expression level of $\mathrm{I} \kappa \mathrm{B} \alpha$ protein was explored in the present study, and we found that $\mathrm{A} \beta$ caused enhanced phosphorylation of $\mathrm{I} \kappa \mathrm{B} \alpha$, which led to the loss of total $\mathrm{I} \kappa \mathrm{B} \alpha$. The reduction of total protein level of $\mathrm{I} \kappa \mathrm{B} \alpha$ enhanced the release of $\mathrm{NF} \kappa \mathrm{B}$ and boosted the level of IL- $1 \beta$ and IL- 6 from astrocytes. However, irisin cotreatment could reduce the $\mathrm{NF} \kappa \mathrm{B}$ level in astrocytes after $A \beta$ stimulation. Moreover, irisin could increase the $\mathrm{I} \kappa \mathrm{B} \alpha$ level and then prevent the release of $\mathrm{NF} \kappa \mathrm{B}$. According to our knowledge so far, our study is the first to show that irisin can exert anti-inflammatory effects on astrocytes in the CNS. Astrocytes have been proved to be the most abundant cell population in the CNS and greatly outnumber neurons [20]. Astrocytes are becoming very important player for the treatment in many diseases and closely involved in memory and cognition deficits in DM [21-23]. Therefore, irisin may be a promising candidate for the treatment of DM, especially in elderly patients. Our finding may not only open a new route for the treatment of $\mathrm{AD}$ but may also provide some evidences to develop novel compounds which target astrocyte in the CNS.

\section{Conclusion}

Our results in this study implied that the neuroprotection of irisin was mediated by blocking the release of Il- $1 \beta$ and IL- 6 from astrocyte instead of its direct action on neurons. Our results also suggested that $\mathrm{NF} \kappa \mathrm{B}$ signaling pathway played an important role in the regulation of irisin on astrocytes exposed to $\mathrm{A} \beta$. While disclosing an important role of astrocytes in $\mathrm{A} \beta$ pathogenesis of $\mathrm{AD}$, our studies also provided new evidence for the idea that irisin might be a promising compound for the treatment $\mathrm{AD}$ and $\mathrm{DM}$.

\section{Data Availability}

The data used to support the findings of this study are available from the corresponding author upon request.

\section{Conflicts of Interest}

The authors declare that they have no conflicts of interest.

\section{Acknowledgments}

This study was supported by a grant from the Shandong Medical and Health Technology Development Project (China, Grant no. 2009QW008) to Dr. Yu Sun.

\section{References}

[1] J. J. Li, G. Dolios, R. Wang, and F. F. Liao, "Soluble betaamyloid peptides, but not insoluble fibrils, have specific effect on neuronal microRNA expression," PLoS One, vol. 9, no. 3, article e90770, 2014.

[2] H. Takuma, T. Tomiyama, K. Kuida, and H. Mori, “Amyloid beta peptide-induced cerebral neuronal loss is mediated by caspase-3 in vivo," Journal of Neuropathology and Experimental Neurology, vol. 63, no. 3, pp. 255-261, 2004.

[3] C. K. Combs, J. C. Karlo, S. C. Kao, and G. E. Landreth, "Betaamyloid stimulation of microglia and monocytes results in TNF $\alpha$-dependent expression of inducible nitric oxide synthase and neuronal apoptosis," The Journal of Neuroscience, vol. 21, no. 4, pp. 1179-1188, 2001.

[4] A. Nazem, R. Sankowski, M. Bacher, and Y. al-Abed, "Rodent models of neuroinflammation for Alzheimer's disease," Journal of Neuroinflammation, vol. 12, no. 1, p. 74, 2015.

[5] V. Rothhammer and F. J. Quintana, "Control of autoimmune CNS inflammation by astrocytes," Seminars in Immunopathology, vol. 37, no. 6, pp. 625-638, 2015.

[6] J. Qiao, J. Wang, H. Wang et al., "Regulation of astrocyte pathology by fluoxetine prevents the deterioration of Alzheimer phenotypes in an APP/PS1 mouse model," Glia, vol. 64, no. 2, pp. 240-254, 2016.

[7] S. Benedini, E. Dozio, P. L. Invernizzi et al., "Irisin: a potential link between physical exercise and metabolism - an observational study in differently trained subjects, from elite athletes to sedentary people," Journal of Diabetes Research, vol. 2017, Article ID 1039161, 7 pages, 2017. 
[8] J. Janson, T. Laedtke, J. E. Parisi, P. O'Brien, R. C. Petersen, and P. C. Butler, "Increased risk of type 2 diabetes in Alzheimer disease," Diabetes, vol. 53, no. 2, pp. 474-481, 2004.

[9] C. D. Wrann, "FNDC5/irisin - their role in the nervous system and as a mediator for beneficial effects of exercise on the brain," Brain Plasticity, vol. 1, no. 1, pp. 55-61, 2015.

[10] A. C. H. Yu, A. Schousboe, and L. Hertz, "Influence of pathological concentrations of ammonia on metabolic fate of ${ }^{14} \mathrm{C}$-labeled glutamate in astrocytes in primary cultures," Journal of Neurochemistry, vol. 42, no. 2, pp. 594-597, 1984.

[11] X. Yang and C. Tohda, "Diosgenin restores $A \beta$-induced axonal degeneration by reducing the expression of heat shock cognate 70 (HSC70)," Scientific Reports, vol. 8, no. 1, article 11707, 2018

[12] H. S. Moon, F. Dincer, and C. S. Mantzoros, "Pharmacological concentrations of irisin increase cell proliferation without influencing markers of neurite outgrowth and synaptogenesis in mouse H19-7 hippocampal cell lines," Metabolism, vol. 62, no. 8, pp. 1131-1136, 2013.

[13] J. Wang, S. Zhu, H. Wang et al., “Astrocyte-dependent protective effect of quetiapine on GABAergic neuron is associated with the prevention of anxiety-like behaviors in aging mice after long-term treatment," Journal of Neurochemistry, vol. 130, no. 6, pp. 780-789, 2014.

[14] A. A. Babcock, L. Ilkjær, B. H. Clausen et al., "Cytokineproducing microglia have an altered beta-amyloid load in aged APP/PS1 Tg mice," Brain, Behavior, and Immunity, vol. 48, pp. 86-101, 2015.

[15] N. Wu, Y. L. Siow, and K. O, "Induction of hepatic cyclooxygenase-2 by hyperhomocysteinemia via nuclear factor- $\kappa \mathrm{B}$ activation," American Journal of Physiology. Regulatory, Integrative and Comparative Physiology, vol. 297, no. 4, pp. R1086-R1094, 2009.

[16] M. Xia, X. Cheng, R. Yi, D. Gao, and J. Xiong, "The binding receptors of $\mathrm{A} \beta$ : an alternative therapeutic target for Alzheimer's disease," Molecular Neurobiology, vol. 53, no. 1, pp. 455-471, 2016.

[17] J. Wang, Y. Zhang, H. Xu et al., "Fluoxetine improves behavioral performance by suppressing the production of soluble $\beta$-amyloid in APP/PS1 mice," Current Alzheimer Research, vol. 11, no. 7, pp. 672-680, 2014.

[18] T. A. Samad, K. A. Moore, A. Sapirstein et al., "Interleukin-1 $\beta$ mediated induction of Cox- 2 in the CNS contributes to inflammatory pain hypersensitivity," Nature, vol. 410, no. 6827 , pp. $471-475,2001$.

[19] F. D'Acquisto, T. Iuvone, L. Rombolà, L. Sautebin, M. di Rosa, and R. Carnuccio, "Involvement of NF- $\kappa$ B in the regulation of cyclooxygenase-2 protein expression in LPS-stimulated J774 macrophages," FEBS Letters, vol. 418, no. 1-2, pp. 175-178, 1997.

[20] S. Jäkel and L. Dimou, "Glial cells and their function in the adult brain: a journey through the history of their ablation," Frontiers in Cellular Neuroscience, vol. 11, p. 24, 2017.

[21] C. Finsterwald, P. Magistretti, and S. Lengacher, "Astrocytes: new targets for the treatment of neurodegenerative diseases," Current Pharmaceutical Design, vol. 21, no. 25, pp. 35703581, 2015.
[22] S. A. Wrighten, G. G. Piroli, C. A. Grillo, and L. P. Reagan, “A look inside the diabetic brain: contributors to diabetes-induced brain aging," Biochimica et Biophysica Acta (BBA) - Molecular Basis of Disease, vol. 1792, no. 5, pp. 444-453, 2009.

[23] A. I. Duarte, E. Candeias, S. C. Correia et al., "Crosstalk between diabetes and brain: glucagon-like peptide-1 mimetics as a promising therapy against neurodegeneration," Biochimica et Biophysica Acta, vol. 1832, no. 4, pp. 527-541, 2013. 


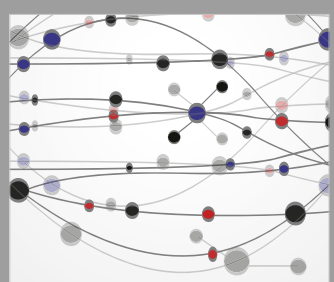

The Scientific World Journal
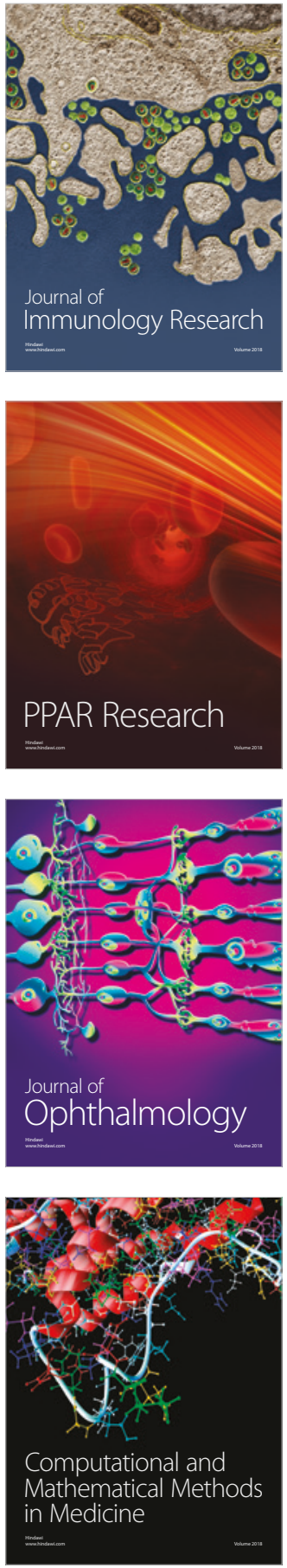

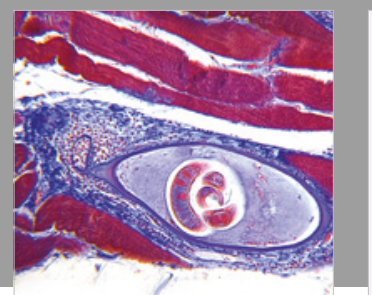

Gastroenterology Research and Practice

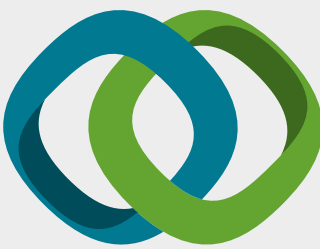

\section{Hindawi}

Submit your manuscripts at

www.hindawi.com
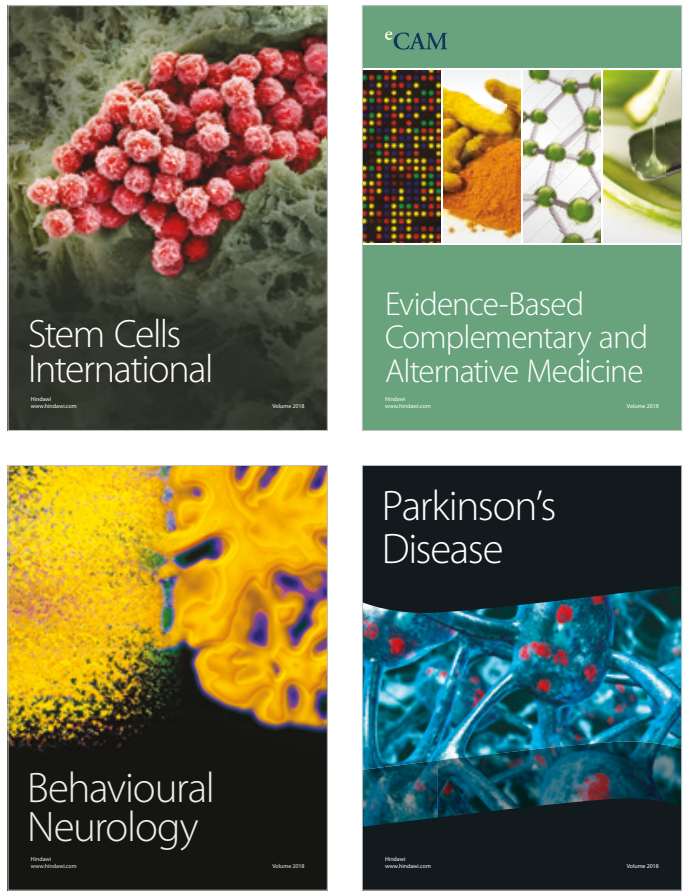

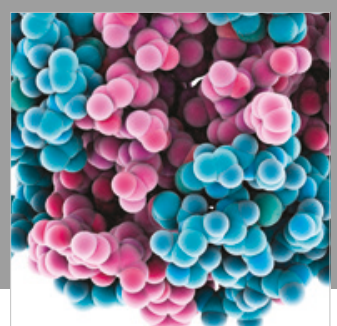

ournal of

Diabetes Research

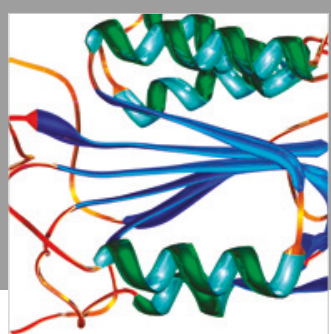

Disease Markers
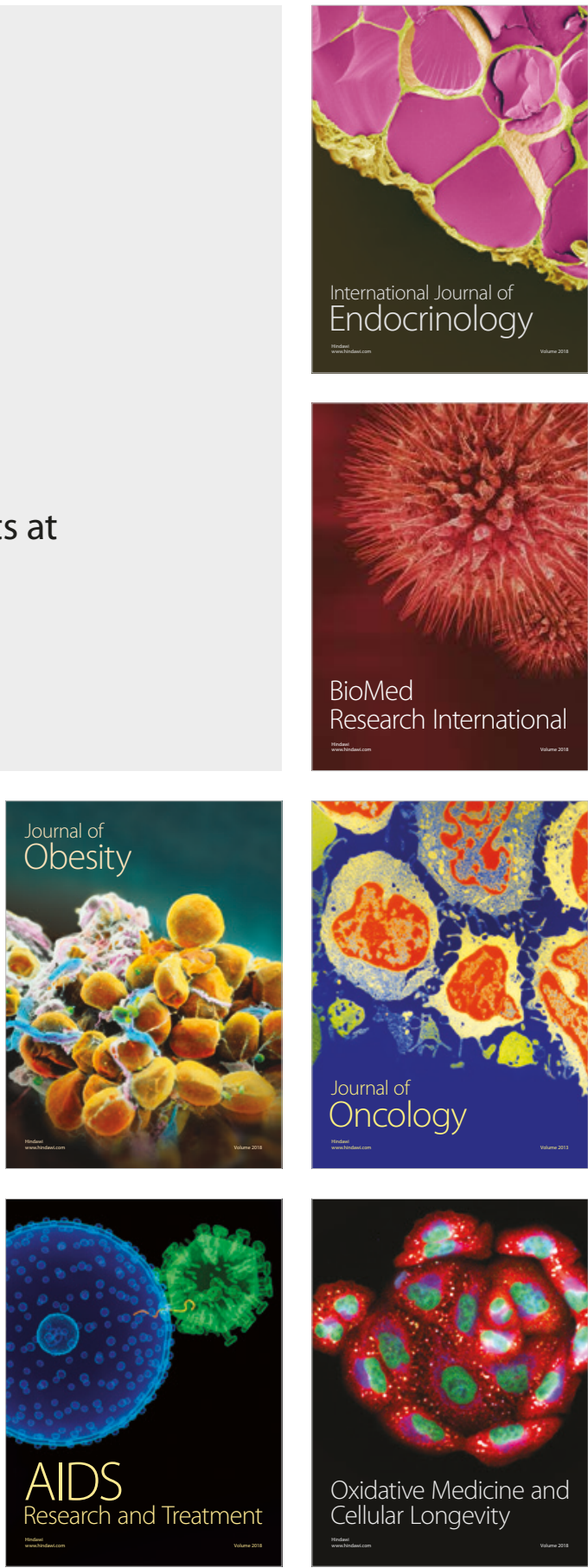